\title{
High expression of S100A12 on intratumoral stroma cells indicates poor prognosis following surgical resection of hepatocellular carcinoma
}

\author{
HAO CAI*, BO-GEN YE* , JIAN-YANG AO, XIAO-DONG ZHU, YUAN-YUAN ZHANG, \\ ZONG-TAO CHAI, CHENG-HAO WANG and HUI-CHUAN SUN \\ Liver Cancer Institute and Zhongshan Hospital, Fudan University, Shanghai 200032, P.R. China
}

Received May 6, 2016; Accepted June 2, 2017

DOI: $10.3892 / \mathrm{ol} .2018 .9270$

\begin{abstract}
The S100 protein family is widely involved in the pathological process of various types of cancer. However, the prognostic value of the S100 protein family member S100A12 in hepatocellular carcinoma (HCC) remains unknown. A total of 139 patients undergoing curative surgical resection for HCC from December 2005 to June 2006 were investigated. Immunohistochemistry of S100A12 tissue was performed and expression was classified according to the total positive staining area. Co-expression of S100A12 with cluster of differentiation $(\mathrm{CD}) 11 \mathrm{~B}, \mathrm{CD} 15$ and CD68 was evaluated using immunofluorescence. Associations between S100A12 expression and preoperative clinicopathological parameters were assessed using a $\chi^{2}$ test or independent sample Student's t-test. Kaplan-Meier estimator survival analysis and multivariate Cox's proportional hazard regression model were used to evaluate the prognostic value of S100A12 expression. The expression of S100A12 was restricted exclusively to stroma cells, primarily to myeloid-derived immune cells, CD15-positive neutrophils and CD68-positive macrophages in particular. A total positive staining area of $1,600 \mu \mathrm{m}^{2}$ was selected as the threshold between high and low S100A12 expression. There was a statistically significant association between intratumoral S100A12 expression and tumor differentiation $(\mathrm{P}=0.010)$. High expression of S100A12 on intratumoral stroma cells was an independent prognostic factor for the overall $(\mathrm{P}=0.002)$ and disease-free survival $(\mathrm{P}=0.007)$ rates of HCC following curative surgical resection. No significant association was identified between peritumoral S100A12
\end{abstract}

Correspondence to: Professor Hui-Chuan Sun, Liver Cancer Institute and Zhongshan Hospital, Fudan University, 180 Fenglin Road, Shanghai 200032, P.R. China

E-mail: sun.huichuan@zs-hospital.sh.cn

*Contributed equally

Key words: hepatocellular carcinoma, S100A12, surgical resection, prognosis expression and HCC prognosis. The results of the present study demonstrated that high expression of S100A12 on intratumoral stroma cells is associated with poor HCC prognosis following curative resection, which may serve as a potential target for an adjuvant therapy.

\section{Introduction}

Hepatocellular carcinoma (HCC) is the sixth most common type of cancer and is the third leading cause of cancer-associated mortality worldwide (1). According to the guidelines of the American Association for the Study of Liver Diseases, surgical resection, liver transplantation and radiofrequency ablation (RFA) are three curative treatment options for HCC (2). However, as the majority of patients are asymptomatic at early stages, only a small portion of patients are amenable to curative treatments. Despite the advances in technology and surgical treatment in recent years, the prognosis of patients with HCC following curative surgical resection remains poor, with a relatively high post-operative recurrence rate (3-5). The molecular mechanisms underlying the recurrence of HCC are not well-understood. However, previous studies have demonstrated that inflammation serves an important role in the development, progression and metastasis of $\mathrm{HCC}$, with a variety of inflammatory cells and mediators identified within the HCC microenvironment (6-8). Myeloid-derived innate immune cells, particularly macrophages and neutrophils, serve important roles in the maintenance of the tumor microenvironment (9).

The S100 protein family belongs to a group of inflammatory molecules which are primarily composed of calcium-binding proteins and have been identified to be involved in a range of pathological processes including infections, cardiovascular diseases and malignancies. There are $\sim 25$ members of the S100 protein family have been identified to date, the majority of which are located on chromosome 1q21 which is frequently associated with genomic rearrangement, forming the genetic background of tumor progression (10). An association between S100 proteins and various types of cancer has been revealed in multiple studies $(10,11)$. Of the $\sim 25$ members, S100A8, S100A9 and S100A12 belong to the calgranulin S100 protein subfamily. Calgranulins are widely expressed 
on myeloid-derived immune cells, particularly on neutrophils and monocytes/macrophages $(12,13)$. It has been reported that S100A8 and S100A9, which usually form heterodimers with each other, are involved in the development of liver malignancies (14-16). In contrast with S100A8 and S100A9, S100A12 functions as a homodimer. Funk et al (17) demonstrated the association between high expression levels of S100A12 on tumor cells with favorable prognosis for oropharyngeal squamous cell carcinoma. However, whether S100A12 is associated with the prognosis of HCC remains unknown. The present study investigated the prognostic value of S100A12 in HCC following curative surgical resections.

\section{Materials and methods}

Study populations. A total of 139 patients diagnosed with HCC who underwent curative surgical resection in Zhongshan Hospital (Fudan University, Shanghai, China) between December 2005 and June 2006 were enrolled in the present study. Patient inclusion required the fulfilment of the following criteria: i) Received curative surgical resection without gross residual tumor; ii) preoperative liver functions classified as Child-Pugh A (18); and iii) histopathological examination confirmed HCC diagnosis. Patients were routinely followed up every 2 months during the first postoperative year and every 3-4 months thereafter (19). The median follow-up time was 34.33 months in the present study. Tumor specimens were prepared for the construction of tissue microarrays according to a previously published protocol (20). Patient characteristics are presented in Table I. The present study was approved by the Zhongshan Hospital Research Ethics Committee and written informed consent was obtained from all patients.

Immunohistochemistry. Tumor tissues were fixed in $10 \%$ neutral buffered formalin at room temperature for 12-24 h. Paraffin embedded tumor tissues were sliced into sections with a thickness of $5 \mu \mathrm{m}$. Paraffin-embedded tumor sections were incubated in $10 \% \mathrm{H}_{2} \mathrm{O}_{2}$ for $15 \mathrm{~min}$ to minimize the non-specific staining due to endogenous peroxidase prior to being incubated in $10 \mathrm{mM}$ citrate buffer ( $\mathrm{pH}$ 6.0) for $30 \mathrm{~min}$ in a steam-cooker for antigen retrieval. Non-specific background staining was blocked with $10 \%$ goat serum (Shanghai Haoran Biological Technology Co., Ltd., Shanghai, China) for $30 \mathrm{~min}$ and subsequently primary mouse anti-S100A12 (1:200; cat. no. LS-C335557; LifeSpan BioSciences, Inc., Seattle, WA, USA), mouse anti-cluster of differentiation (CD)15 (1:100; cat. no. ab754; Abcam, Cambridge, UK) and mouse anti-CD68 (1:100; cat. no. ab49777; Abcam) antibodies were applied for incubation overnight at $4^{\circ} \mathrm{C}$, according to the manufacturer's protocol. Subsequently, sections were incubated with ready-to-use horseradish peroxidase (HRP)-conjugated anti-mouse IgG (HRP Polymer Quanto; cat. no. TL-015-QHD; Lab Vision Corporation, Fremont, CA, USA) at room temperature for $30 \mathrm{~min}$ followed by incubation with mixture of DAB Quanto Substrate (cat. no. TL-015-QHD; Lab Vision Corporation) and DAB Quanto Chromogen (cat. no. TL-015-QHD; Lab Vision Corporation) at a ratio of 1,000:30 at room temperature for 1-5 min.

For immunofluorescence staining, frozen tumor sections were blocked with $10 \%$ donkey serum (Jackson
Table I. Basic clinicopathological parameters of included patients.

\begin{tabular}{|c|c|c|c|}
\hline \multirow[b]{2}{*}{ Characteristics } & \multicolumn{2}{|c|}{$\begin{array}{c}\text { Intratumoral S100A12 } \\
\text { expression }\end{array}$} & \multirow[b]{2}{*}{ P-value } \\
\hline & Low & High & \\
\hline Age, years & $52.31 \pm 11.74$ & $51.12 \pm 8.89$ & 0.573 \\
\hline Sex & & & 0.759 \\
\hline Male & 98 & 21 & \\
\hline Female & 16 & 4 & \\
\hline HBsAg & & & 0.526 \\
\hline Negative & 17 & 2 & \\
\hline Positive & 97 & 23 & \\
\hline Concurrent cirrhosis & & & 0.832 \\
\hline No & 89 & 20 & \\
\hline Yes & 25 & 5 & \\
\hline AFP, ng/ml & & & 0.726 \\
\hline$\leq 20$ & 36 & 7 & \\
\hline$>20$ & 78 & 18 & \\
\hline Tumor size, $\mathrm{cm}$ & $5.60 \pm 3.64$ & $5.68 \pm 4.21$ & 0.924 \\
\hline Tumor number & & & 0.765 \\
\hline Solitary & 95 & 22 & \\
\hline Multiple & 19 & 3 & \\
\hline Tumor encapsulation & & & 0.060 \\
\hline No & 55 & 7 & \\
\hline Yes & 59 & 18 & \\
\hline Vascular tumor thrombus & & & 0.383 \\
\hline No & 66 & 12 & \\
\hline Yes & 48 & 13 & \\
\hline Tumor differentiation & & & $0.010^{\mathrm{a}}$ \\
\hline I-II & 88 & 13 & \\
\hline III-IV & 26 & 12 & \\
\hline TNM stage & & & 0.549 \\
\hline I-II & 97 & 20 & \\
\hline III-IV & 17 & 5 & \\
\hline
\end{tabular}

${ }^{\mathrm{a} P}<0.05$ by $\chi^{2}$ test. HBsAg, hepatitis $\mathrm{B}$ surface antigen; AFP, $\alpha$-fetoprotein; TNM, tumor-node-metastasis.

ImmunoResearch Laboratories, Inc., West Grove, PA, USA) for at room temperature for $30 \mathrm{~min}$ before incubation with primary rabbit anti-S100A12 antibody (1:100; cat. no. LS-C335557, LifeSpan BioSciences, Inc.) together with mouse anti-CD11B (1:100, cat. no. ab34216, Abcam), mouse anti-CD15 (1:100, cat. no. ab754, Abcam) or mouse anti-CD68 (1:100, cat. no. ab53444, Abcam) antibody overnight at $4^{\circ} \mathrm{C}$. Sections were washed three times in PBS followed by incubation with Alexa Fluor 546-conjugated anti-rabbit IgG (1:250; cat. no. A10040; Invitrogen; Thermo Fisher Scientific, Inc., Waltham, MA, USA) and Alexa Fluor 488-conjugated anti-mouse IgG (1:250; cat. no. A21202; Invitrogen; Thermo Fisher Scientific, Inc.) secondary antibodies for $2 \mathrm{~h}$ at $37^{\circ} \mathrm{C}$. Sections were then stained with $10 \mu \mathrm{g} / \mathrm{ml}$ DAPI for $10 \mathrm{~min}$. 
Sections were washed in PBS and mounted with anti-fade reagent (SouthernBiotech, Birmingham, AL, USA) and observed using a confocal fluorescence microscope $(1,260 \mathrm{X}$ objective magnification; Olympus Corporation, Tokyo, Japan).

Tissue microarray analysis. The assessment of S100A12 expression was performed by a computerized image system comprising a Leica charge-coupled device camera DFC420 connected to a Leica DM IRE2 microscope (Leica Microsystems, Ltd., Milton Keynes, UK). Images of three random 'hotspots' where S100A12 was most populous at magnification $\mathrm{x} 20$, were captured using Leica QWin Plus software (v.3; Leica Microsystems, Ltd.), and the integrated optical total positive staining area (TPSA) of these images was determined using Image-Pro Plus software version 6 (Media Cybernetics, Inc. Rockville, MD, USA). The camera parameter was constant throughout the whole process. An optimal TPSA was selected as a threshold to define the expression of S100A12 as low or high on the basis of the log-rank test as described previously (21). Three experienced researchers, who are experienced in the pathological examination of HCC, reviewed the results independently. Any discrepancies were resolved by discussion in order to reach a consensus.

Statistical analysis. Quantitative variables of normal distribution are presented as the mean \pm standard deviation and compared using the independent sample Student's t-test or one-way analysis of variance test (Tukey post-hoc test) where applicable. Quantitative variables of abnormal distribution are presented as the median and compared with non-parametric tests (Mann Whitney-U test). Qualitative variables are presented as relative frequencies and compared using Pearson $\chi^{2}$ test or Fisher's exact test. The Kaplan-Meir estimator survival analysis using a log-rank test was performed to compare the overall survival (OS) and disease-free survival (DFS) rates following curative surgical resection of HCC between different groups. A multivariate Cox's proportional hazard model was used to assess the association between S100A12 and patient survival rate, together with other confounding factors including tumor size, tumor number, presence of vascular tumor embolus, tumor-node-metastasis (TNM) stage, tumor differentiation and pre-operative serum $\gamma$-glutamyltranspeptidase (GGT). All statistical analysis was performed using the commercially available software SPSS (version 18.0; IBM Corp., Armonk, NY, USA). $\mathrm{P}<0.05$ was considered to indicate a statistically significant difference.

\section{Results}

Patient characteristics and S100A12 expression. A total of 139 patients were included in the present study. Measured by the TPSA, intratumoral S100A12 expression was low in 114 patients (TPSA, $<1,600 \mu \mathrm{m}^{2}$ ) and high in 25 patients (TPSA, $>1,600 \mu \mathrm{m}^{2}$ ). Basic clinicopathological parameters, including age, sex, hepatitis B surface antigen, concurrent cirrhosis, $\alpha$-fetoprotein, tumor size, tumor number, presence of vascular tumor thrombus, TNM stage and tumor Edmonson-Steiner differentiation score (I-IV, with an, increased score indicating a worse differentiation) (22), were collected (Table I).

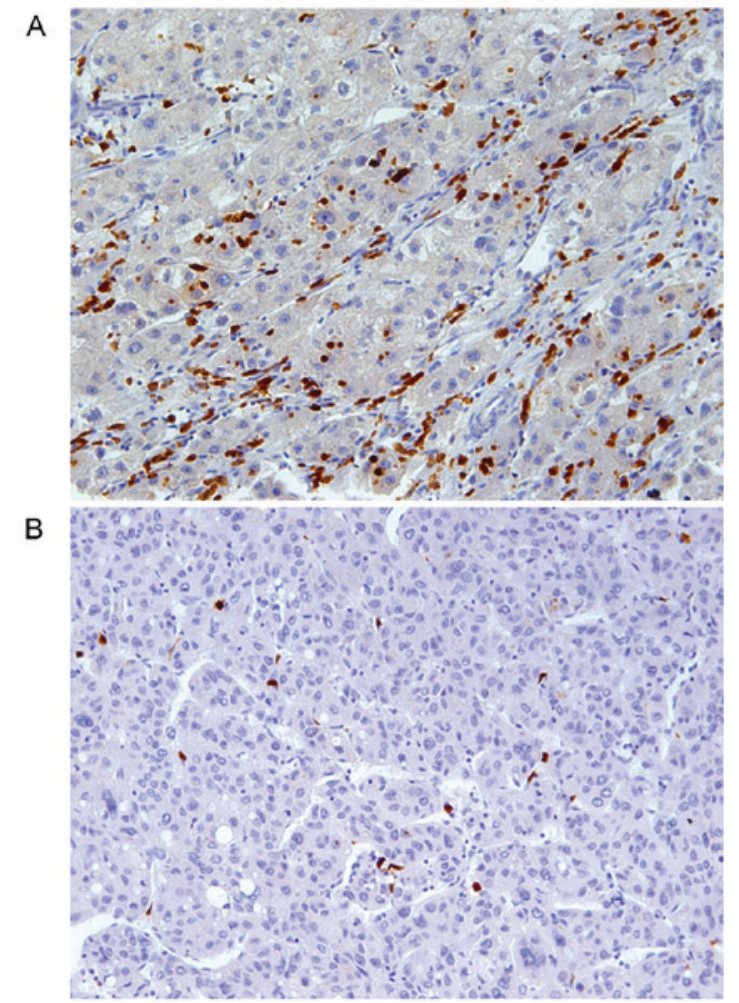

Figure 1. Immunohistochemistry staining (magnification, x200) demonstrated the expression of S100A12 in hepatocellular carcinoma tissues. The S100A12-expressing cells exhibited a relatively smaller size and were scattered within tumor tissues. Intratumoral S100A12 was classified as (A) relatively high or (B) low according to the total positive staining area.

S100A12 was exclusively expressed in the cytoplasm of stroma cells with relatively smaller size compared with the tumor cells (Fig. 1). The expression of S100A12 was significantly decreased $(\mathrm{P}<0.001)$ on intratumoral stroma cells (median TPSA, $270 \mu \mathrm{m}^{2}$ ) compared with peritumoral stroma cells (median TPSA, $836 \mu \mathrm{m}^{2}$ ). Immunofluorescence staining revealed that S100A12 was primarily expressed on CD11B-positive myeloid-derived immune cells particularly CD15-positive neutrophils and CD68-positive macrophages, which serve important roles in the HCC tumor microenvironment (Fig. 2). There was no association between the TPSA of S100A12 and that of CD15 ( $\mathrm{P}=0.603), \mathrm{CD} 68(\mathrm{P}=0.562)$ or the sum of the two $(\mathrm{P}=0.526)$ in 30 of 139 patients. A statistically significant association between S100A12 expression and tumor differentiation was observed upon evaluation with the Edmonson-Steiner score $(\mathrm{P}=0.010)$. Pre-operative serum alanine aminotransferase $(\mathrm{P}=0.539)$, aspartate aminotransferase $(\mathrm{P}=0.595)$, albumin $(\mathrm{P}=0.194)$, bilirubin $(\mathrm{P}=0.601)$, prothrombin time $(\mathrm{P}=0.124)$ and $\mathrm{GGT}(\mathrm{P}=0.401)$, which all reflect the severity of liver damage or the activity of inflammation, were not statistically different between high and low S100A12 expression. There was no association between S100A12 expression and other clinicopathological parameters. There were 67 patients at TNM stage I and 50 at stage II in total. The expression of S100A12 between TNM I and II was not significantly different with a P-value of 0.620 .

Prognostic value of S100A12 expression within tumor tissues. Measured by the TPSA, high expression of S100A12 on 


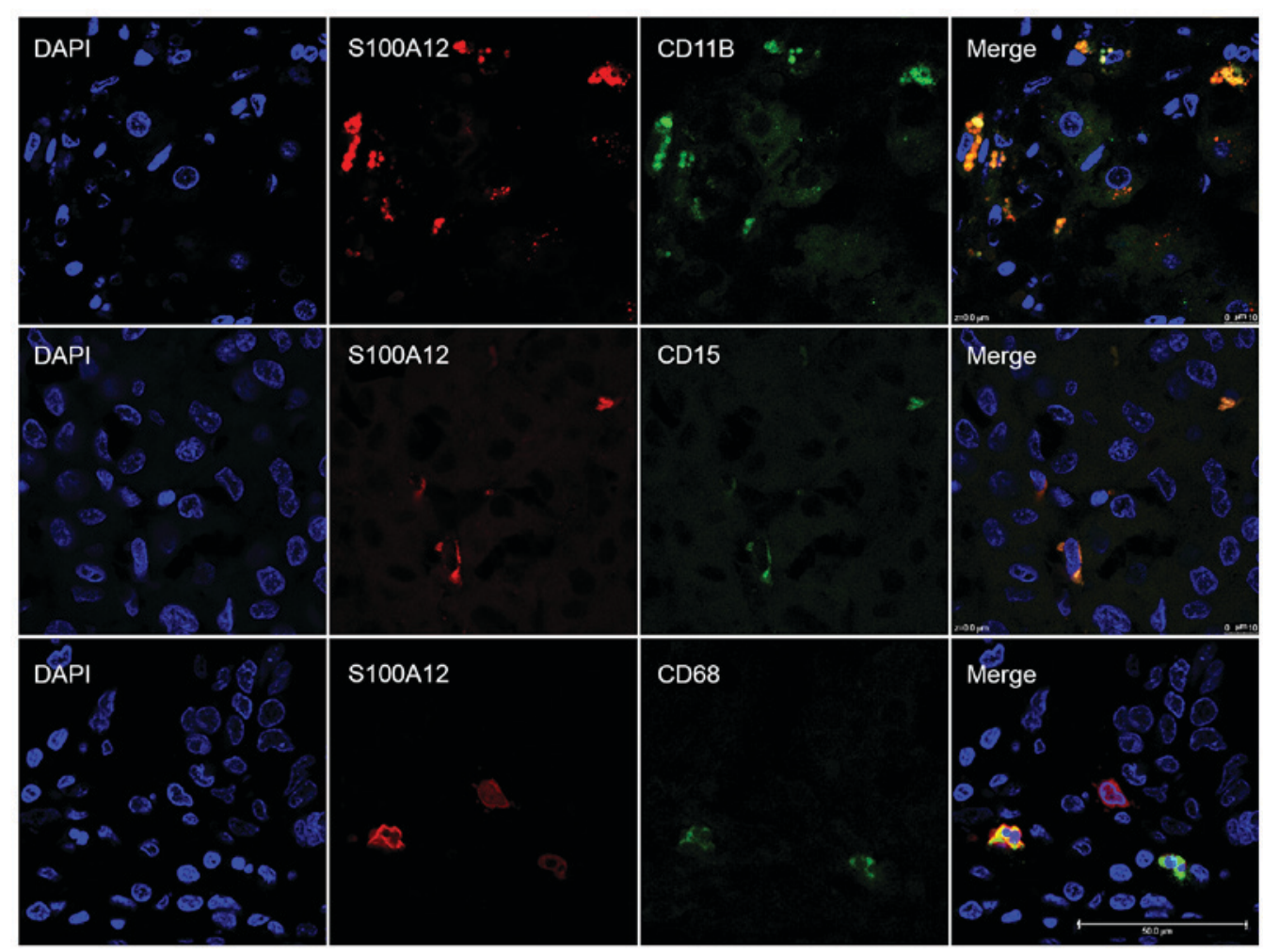

Figure 2. Immunofluorescence staining revealed co-expression of S100A12 with CD11B, CD15 and CD68 in hepatocellular carcinoma tissues. The cell nucleus was stained blue, S100A12 was stained red, and CD11B, CD15 and CD68 were stained green. Scale bar, $50 \mu \mathrm{m}$. CD, cluster of differentiation.

intratumoral stroma cells was associated with a poorer OS rate $\left(\chi^{2}=7.519, \mathrm{P}=0.006\right)$. The 1 -, 2 - and 3-year OS rates were $88.6,75.3$ and $65.8 \%$ for patients with low S100A12 expression, compared with $80.0,48.0$ and $40.0 \%$ for patients with high S100A12 expression. The DFS rate of patients with low expression of S100A12 was significantly increased, with 1-, 2and 3-year DFS rates of 78.6, 64.5 and 54.9\%, compared with 62.0, 34.3 and $17.2 \%$ for patients with high S100A12 expression $\left(\chi^{2}=6.471 ; \mathrm{P}=0.011\right.$; Fig. 3$)$. There were no statistically significant associations between peritumoral S100A12 expression and the OS $(\mathrm{P}=0.709)$ or $\mathrm{DFS}(\mathrm{P}=0.759)$ rate following surgical resection of $\mathrm{HCC}$.

With the exception of S100A12, univariate analysis revealed that tumor size $(\mathrm{P}<0.001)$, tumor number $(\mathrm{P}=0.015)$, presence of vascular tumor thrombus $(\mathrm{P}=0.004)$, tumor differentiation score $(\mathrm{P}=0.006)$, TNM stage $(\mathrm{P}<0.001)$ and pre-operative serum GGT $(\mathrm{P}<0.001)$ were associated with the OS rate of patients with HCC. Tumor size $(\mathrm{P}<0.001)$, presence of vascular tumor thrombus $(\mathrm{P}=0.035)$, TNM stage $(\mathrm{P}=0.003)$ and pre-operative serum GGT $(\mathrm{P}=0.017)$ were also associated with the DFS of patients with HCC. Multivariate Cox's proportional hazard model analysis demonstrated that high expression of S100A12 was an independent prognostic factors for the OS $(\mathrm{P}=0.001)$ and $\mathrm{DFS}(\mathrm{P}=0.007)$ rates of patients with $\mathrm{HCC}$, as presented in Table II.

\section{Discussion}

There are a limited number of studies on S00A12 and its association with cancer incidence. One reason for the limited knowledge about S100A12 may be that S100A12 is constitutionally expressed in humans without an ortholog in rodents. With experimental studies in the field of cancer research now primarily based on model organisms, particularly rodents, this may be a limiting factor.

Consistent with the literature, the results of the present study demonstrated that S100A12 was primarily expressed on myeloid-derived immune cells, including neutrophils and monocytes/macrophages $(12,13)$. The immunohistochemistry results of the present study revealed that S100A12 was not expressed on tumor cells or vascular endothelial cells. Immunofluorescence staining demonstrated that S100A12 was predominantly expressed on CD11B-positive myeloid-derived immune cells, particularly on CD15-positive neutrophils and CD68-positive macrophages. The results of the present study revealed that intratumoral S100A12 served as an independent prognostic factor for HCC following curative surgical resection. High expression of S100A12 on intratumoral stroma cells indicated poor OS and DFS rates following curative surgical resection.

Tumor-associated macrophages and neutrophils have been intensively studied (9) and an association with HCC has been demonstrated. However, a previous study revealed that intratumoral macrophage infiltration was not associated with the OS or DFS rates of HCC following curative surgical resection (20). Kuang et al (23) also demonstrated that intratumoral neutrophils were not associated with the prognosis of HCC. There was no association between S100A12 with CD15 ( $\mathrm{P}=0.603)$, CD68 $(\mathrm{P}=0.562)$ or the two together $(\mathrm{P}=0.526)$. It may be that certain types of macrophages or neutrophils that infiltrate tumor tissues that serve a critical role in the progression of HCC. Erbel et al (24) identified a new type of atherosclerotic 
Table II. Multivariate analysis of factors associated with overall and disease-free survival rates of hepatocellular carcinoma following curative surgical resection.

Overall survival

HR $(95 \% \mathrm{CI}) \quad$ P-value
$\mathrm{HR}(95 \% \mathrm{CI})$
Disease-free survival

Characteristic

$2.81(1.32-5.98)$

$0.007^{\mathrm{a}}$

$1.96(1.03-3.74)$

$0.040^{\mathrm{a}}$

$>5$ vs. $\leq 5$

$1.15(0.42-3.13)$

0.791

NA

NA

Solitary vs. multiple

$0.84(0.42-1.68)$

0.626

$1.12(0.62-2.05)$

0.704

Yes vs. no

$2.00(0.97-4.11)$

0.059

NA

NA

III-IV vs. I-II

$2.36(0.79-7.12)$

0.126

$1.67(0.78-3.56)$

0.188

III-IV vs. I-II

$2.60(1.40-4.82)$

$0.002^{\mathrm{a}}$

$1.70(0.95-3.05)$

0.075

$>91$ vs. $\leq 91$

$3.17(1.53-6.57)$

$0.002^{\mathrm{a}}$

$2.53(1.29-4.96)$

$0.007^{\mathrm{a}}$

S100A12 expression

Low vs. high

${ }^{\mathrm{a}} \mathrm{P}<0.05$. HR, hazard ratio; CI, confidence interval; NA, not adopted; TNM, tumor-node-metastasis; GGT, $\gamma$-glutamyltranspeptidase.
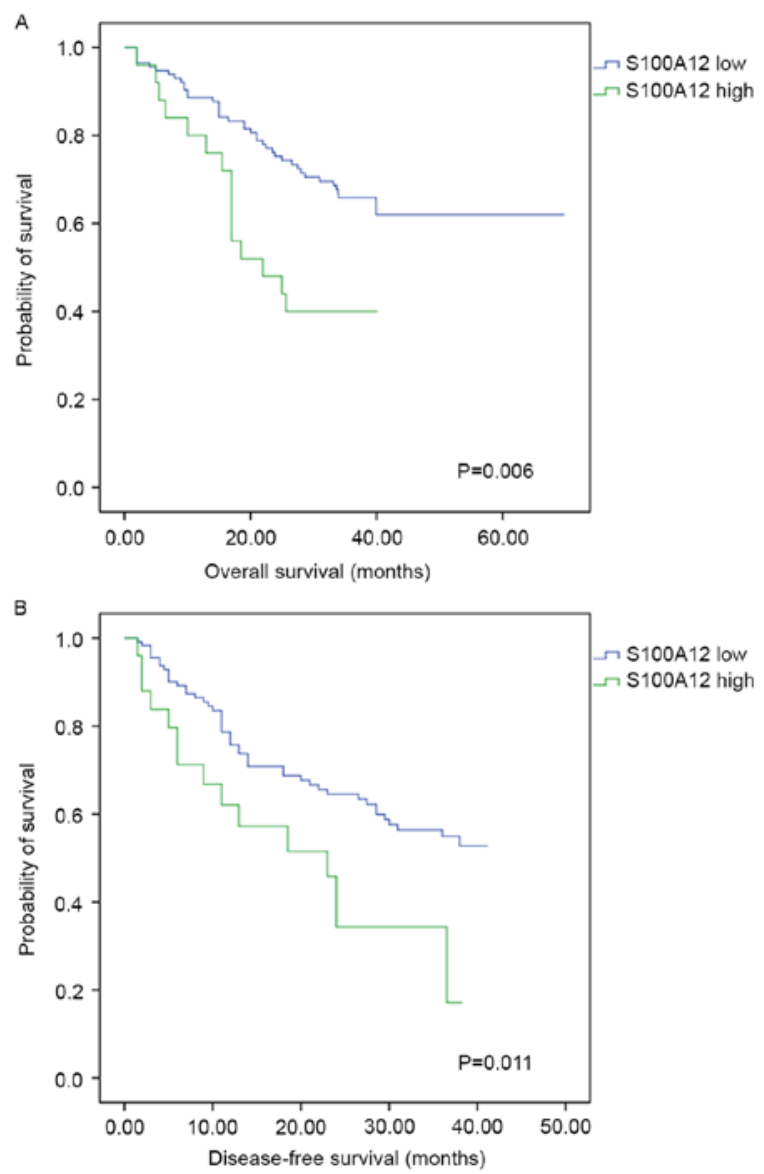

Figure 3. Kaplan-Meier estimator survival curves for hepatocellular carcinoma following curative resection according to intratumoral S100A12 expression. (A) Overall and (B) disease-free survival rates were compared using a two-sided log-rank test. plaque-associated MMP7-positive, S100A8-positive and CD68-positive macrophage termed M4 macrophage, which is distinct from the typical M1 and M2 macrophage type. As an analog of S100A12, S100A8 expression was interleukin 10- and prostaglandin $\mathrm{E}_{2}$-dependent, which suggested anti-inflammatory properties of S100A12-positive macrophages (25). In addition, neutrophils may be involved in the progression of HCC through an S100A12 signaling pathway, an area where further investigation is required. Neutrophils expressing S100A12 may be associated with tumor progression and may account for the post-operative recurrence of HCC. As members of the calgranulins, S100A8 and S100A9 may possess similar functions to those of S100A12. A previous study revealed that S100A8 and S100A9 expression was induced by nuclear factor $\kappa \mathrm{B}$, promoting $\mathrm{HCC}$ progression by activating the reactive oxygen species signaling pathway (16). Additionally, S100A9 alone increased the viability and invasiveness of human HCC cells by activating the mitogen-activated protein kinase signaling pathway (15).

In the present study, tumor size and pre-operative serum GGT were also identified to be independent prognostic factors of post-operative OS rates, and tumor size was identified to be an independent prognostic factor of post-operative DFS rates. These results were consistent with those of previous studies; tumor size was an accepted prognostic factor of HCC following surgical resection (26) and GGT was reported to be associated with poor prognosis following surgical resection, RFA and transcatheter arterial chemoembolization (27-29). Fu et al (27) revealed that high pre-operative serum GGT was associated with poor OS and DFS rates following surgical resection of HCC, which supports the results of the present study. However, the underlying molecular mechanism remains elusive (27). 
The results of the present study revealed an association between S100A12 and poor tumor differentiation, which was in accordance with previous studies (10). Similar results were reported for S100A9 and its association with tumor differentiation in HCC (30). It is known that crosstalk between tumor cells and surrounding immune cells contributes to the progression, invasion and metastasis of tumor cells (9). S100A12 expression in tumor-infiltratory myeloid-derived immune cells may be induced by tumor cells, attracting various inflammatory cells to migrate to the tumor site and exert pro- and anti-inflammation activities by secreting cytokines of diverse functions. Transforming growth factor $\beta 1$ (TGF- $\beta 1$ ) and other inflammatory mediators have been revealed to be involved in the process of epithelial-mesenchymal transition (EMT), which is associated with the dedifferentiation of cancer cells (31-33). Another member of the S100 protein family, S100A4, was reported to upregulate SNAI1, which was downstream of TGF- $\beta 1$, serving a role in the EMT of HCC cells (34). As with S100A4, a similar process may explain the association between S100A12 and poor tumor differentiation during the course of HCC progression.

There were a number of limitations to the present study. First, S100A12 is unique to humans and is expressed on multiple myeloid-derived immune cells including neutrophils and monocytes/macrophages, which impeded further knowledge of the underlying molecular mechanisms by which S100A12 influences HCC progression and recurrence. Secondly, as the expression of S100A12 within HCC tissues was low in general, there was only a small portion of patients with high intratumoral S100A12 expression. The sample size of the high-S100A12 group was relatively small which could limit the reliability of results. Further studies including incorporating more eligible patients are required.

The present study revealed that S100A12 may provide a potential target for the immune therapy of HCC. S100A12 may be a potential target for antitumor treatment. Also, the decrease in S100A12 expression may be a marker of clinical effectiveness of immune therapy for HCC.

\section{Acknowledgements}

The authors would like to Mrs Ke Qiao from Key Laboratory of Medical Molecular virology, Ministry of Education and Public Health, School of Basic Medical Sciences, Fudan University (Shanghai, China) for her technical expertise in microscopic imaging techniques.

\section{Funding}

The collection, analysis and interpretation of data and manuscript writing were supported by grants from the National Natural Science Foundation of China (grant nos. 81372655 and 81472224) and the National Key Basic Research Program [(973 project; grant no. 2015CB554005) from the Ministry of Science and Technology of China.

\section{Availability of data and materials}

The datasets used and/or analyzed during the current study are available from the corresponding author on reasonable request.

\section{Authors' contributions}

HS and HC designed the study. HC contributed to the writing of the manuscript. HC and BY analyzed the clinicopathological data and performed the survival analysis. $\mathrm{HC}$ and $\mathrm{BY}$ performed the immunohistochemistry staining of the tissue microarray. $\mathrm{HC}$ and $\mathrm{XZ}$ performed the immunofluorescence staining of HCC frozen sections. YZ, ZC and CW collected the clinicopathological data and follow-up information of patients with HCC. All authors read and approved the final manuscript.

\section{Ethics approval and consent to participate}

The present study was approved by the Zhongshan Hospital Research Ethics Committee. Written informed consent for the use of their tissue and clinicopathologic data was obtained from all patients.

\section{Patient consent for publication}

There is no patient consent for publication. However, all identifying information has been removed in the present study.

\section{Competing interests}

The authors declare that they have no competing interests.

\section{References}

1. Global Burden of Disease Cancer Collaboration; Fitzmaurice C, Dicker D, Pain A, Hamavid H, Moradi-Lakeh M, MacIntyre MF, Allen C, Hansen G, Woodbrook R, et al: The global burden of cancer 2013. JAMA Oncol 1: 505-527, 2015.

2. Bruix J and Sherman M; American Association for the Study of Liver Diseases: Management of hepatocellular carcinoma: An update. Hepatology 53: 1020-1022, 2011.

3. Roayaie S, Obeidat K, Sposito C, Mariani L, Bhoori S, Pellegrinelli A, Labow D, Llovet JM, Schwartz $M$ and Mazzaferro V: Resection of hepatocellular cancer $</=2 \mathrm{~cm}$ : Results from two Western centers. Hepatology 57: 1426-1435, 2013.

4. Shrager B, Jibara G, Schwartz M and Roayaie S: Resection of hepatocellular carcinoma without cirrhosis. Ann Surg 255: 1135-1143, 2012.

5. Hasegawa K, Kokudo N, Imamura H, Matsuyama Y, Aoki T, Minagawa M, Sano K, Sugawara Y, Takayama T and Makuuchi M: Prognostic impact of anatomic resection for hepatocellular carcinoma. Ann Surg 242: 252-259, 2005.

6. Alison MR, Nicholson LJ and Lin WR: Chronic inflammation and hepatocellular carcinoma. Recent Results Cancer Res 185: 135-148, 2011.

7. Hu B, Yang XR, Xu Y, Sun YF, Sun C, Guo W, Zhang X, Wang WM, Qiu SJ, Zhou J and Fan J: Systemic immune-inflammation index predicts prognosis of patients after curative resection for hepatocellular carcinoma. Clin Cancer Res 20: 6212-6222, 2014.

8. Huang J, Xu L, Luo Y, He F, Zhang Y and Chen M: The inflammation-based scores to predict prognosis of patients with hepatocellular carcinoma after hepatectomy. Med Oncol 31: 883, 2014.

9. Galdiero MR, Bonavita E, Barajon I, Garlanda C, Mantovani A and Jaillon S: Tumor associated macrophages and neutrophils in cancer. Immunobiology 218: 1402-1410, 2013.

10. Chen H, Xu C, Jin Q and Liu Z: S100 protein family in human cancer. Am J Cancer Res 4: 89-115, 2014.

11. Salama I, Malone PS, Mihaimeed F and Jones JL: A review of the S100 proteins in cancer. Eur J Surg Oncol 34: 357-364, 2008.

12. Goyette J and Geczy CL: Inflammation-associated S100 proteins: New mechanisms that regulate function. Amino Acids 41: 821-842, 2011. 
13. Gross SR, Sin CG, Barraclough R and Rudland PS: Joining S100 proteins and migration: for better or for worse, in sickness and in health. Cell Mol Life Sci 71: 1551-1579, 2014.

14. Wu R, Duan L, Cui F, Cao J, Xiang Y, Tang Y and Zhou L: S100A9 promotes human hepatocellular carcinoma cell growth and invasion through RAGE-mediated ERK1/2 and p38 MAPK pathways. Exp Cell Res 334: 228-238, 2015.

15. Wu R, Duan L, Ye L, Wang H, Yang X, Zhang Y, Chen X, Zhang Y, Weng Y, Luo J, et al: S100A9 promotes the proliferation and invasion of HepG2 hepatocellular carcinoma cells via the activation of the MAPK signaling pathway. Int J Oncol 42: 1001-1010, 2013.

16. Nemeth J, Stein I, Haag D, Riehl A, Longerich T, Horwitz E, Breuhahn K, Gebhardt C, Schirmacher P, Hahn M, et al: S100A8 and S100A9 are novel nuclear factor kappa B target genes during malignant progression of murine and human liver carcinogenesis. Hepatology 50: 1251-1262, 2009.

17. Funk S, Mark R, Bayo P, Flechtenmacher C, Grabe N, Angel P, Plinkert PK and Hess J: High S100A8 and S100A12 protein expression is a favorable prognostic factor for survival of oropharyngeal squamous cell carcinoma. Int J Cancer 136: 2037-2046, 2015.

18. European Association for the Study of the Liver. Electronic address: easloffice@easloffice.eu; European Association for the Study of the Liver: EASL Clinical Practice Guidelines: Management of hepatocellular carcinoma. J Hepatol 69: 182-236, 2018.

19. Sun HC, Zhang W, Qin LX, Zhang BH, Ye QH, Wang L, Ren N, Zhuang PY, Zhu XD, Fan J and Tang ZY: Positive serum hepatitis $\mathrm{B}$ e antigen is associated with higher risk of early recurrence and poorer survival in patients after curative resection of hepatitis B-related hepatocellular carcinoma. J Hepatol 47: 684-690, 2007.

20. Zhu XD, Zhang JB, Zhuang PY, Zhu HG, Zhang W, Xiong YQ Wu WZ, Wang L, Tang ZY and Sun HC: High expression of macrophage colony-stimulating factor in peritumoral liver tissue is associated with poor survival after curative resection of hepatocellular carcinoma. J Clin Oncol 26: 2707-2716, 2008

21. Budczies J, Klauschen F, Sinn BV, Győrffy B, Schmitt WD, Darb-Esfahani S and Denkert C: Cutoff Finder: A comprehensive and straightforward Web application enabling rapid biomarker cutoff optimization. PLoS One 7: e51862, 2012.

22. Liu GJ, Xu HX, Lu MD, Xie XY, Xu ZF, Zheng YL and Liang JY: Correlation between enhancement pattern of hepatocellular carcinoma on real-time contrast-enhanced ultrasound and tumour cellular differentiation on histopathology. Br J Radiol 80: 321-330, 2007.

23. Kuang DM, Zhao Q, Wu Y, Peng C, Wang J, Xu Z, Yin XY and Zheng L: Peritumoral neutrophils link inflammatory response to disease progression by fostering angiogenesis in hepatocellular carcinoma. J Hepatol 54: 948-955, 2011.
24. Erbel C, Tyka M, Helmes CM, Akhavanpoor M, Rupp G, Domschke G, Linden F, Wolf A, Doesch A, Lasitschka F, et al: CXCL4-induced plaque macrophages can be specifically identified by co-expression of MMP7+S100A8+ in vitro and in vivo. Innate Immu 21: 255-265, 2015.

25. Hsu K, Chung YM, Endoh Y and Geczy CL: TLR9 ligands induce S100A8 in macrophages via a STAT3-dependent pathway which requires IL-10 and PGE2. PLoS One 9: e103629, 2014

26. Schlachterman A, Craft WW Jr, Hilgenfeldt E, Mitra A and Cabrera R: Current and future treatments for hepatocellular carcinoma. World J Gastroenterol 21: 8478-8491, 2015.

27. Fu S, Guo Z, Li S, Kuang M, Hu W, Hua Y, He X and Peng B: Prognostic value of preoperative serum gamma-glutamyltranspeptidase in patients with hepatocellular carcinoma after hepatectomy. Tumour Biol 37: 3433-3440, 2016.

28. Ma H, Zhang L, Tang B, Wang Y, Chen R, Zhang B, Chen Y, Ge N, Wang Y, Gan Y, et al: $\gamma$-Glutamyltranspeptidase is a prognostic marker of survival and recurrence in radiofrequency-ablation treatment of hepatocellular carcinoma. Ann Surg Oncol 21: 3084-3089, 2014

29. Zhang JB, Chen Y, Zhang B, Xie X, Zhang L, Ge N, Ren Z and Ye SL: Prognostic significance of serum gamma-glutamyl transferase in patients with intermediate hepatocellular carcinoma treated with transcatheter arterial chemoembolization. Eur J Gastroenterol Hepatol 23: 787-793, 2011.

30. Arai K, Yamada T and Nozawa R: Immunohistochemical investigation of migration inhibitory factor-related protein (MRP)-14 expression in hepatocellular carcinoma. Med Oncol 17: 183-188, 2000.

31. Pang MF, Georgoudaki AM, Lambut L, Johansson J, Tabor V, Hagikura K, Jin Y, Jansson M, Alexander JS, Nelson CM, et al: TGF- $\beta 1$-induced EMT promotes targeted migration of breast cancer cells through the lymphatic system by the activation of CCR7/CCL21-mediated chemotaxis. Oncogene 35: 748-760, 2016.

32. Ricciardi M, Zanotto M, Malpeli G, Bassi G, Perbellini O, Chilosi M, Bifari F and Krampera M: Epithelial-to-mesenchymal transition (EMT) induced by inflammatory priming elicits mesenchymal stromal cell-like immune-modulatory properties in cancer cells. Br J Cancer 112: 1067-1075, 2015.

33. van Zijl F, Zulehner G, Petz M, Schneller D, Kornauth C, Hau M, Machat G, Grubinger M, Huber H and Mikulits W: Epithelial-mesenchymal transition in hepatocellular carcinoma. Future Oncol 5: 1169-1179, 2009.

34. Zheng X, Gai X, Wu Z, Liu Q and Yao Y: Metastasin leads to poor prognosis of hepatocellular carcinoma through partly inducing EMT. Oncol Rep 29: 1811-1818, 2013. 